\title{
Research on Competitive Transformation of Chinese Martial Arts
}

\author{
Ran Li \\ Xi'an Aeronautical University, Xi'an, 710077, China
}

\author{
Keywords: China, Martial Arts, Competitive Transformation
}

\begin{abstract}
With the development and transformation of society, Chinese martial arts also keep transforming to enter modernization process and blend in world sports gradually. Martial arts develop into competitive pattern gradually and competitive martial arts developed from traditional martial arts. Developing modern Chinese martial arts is the inexorable trend of times, but competitive Chinese martial art is not the affiliation that walks to the world and future. The competitiveness of traditional martial arts gets a certain development after depression, burgeon and exploration, which makes competitive transformation possess certain obvious styles and provide power for the development of Chinese martial arts.
\end{abstract}

\section{Introduction}

China suffers from very serious and direct cultural shocks due to the development of economic globalization. People start to realize the difference between themselves and the West. They start to refer to Western model gradually and develop methods which are suitable for their own features. China enters the period of social transformation gradually, which is embodied specifically in all systems and layers of society. Chinese martial arts belong to the essence and representative of national traditional sports, the development of Western culture brings large shocks to Chinese traditional martial arts. Chinese martial arts also enter transformation period with the progress and transformation of society. The important trend of modern development of Chinese martial arts is the competitiveness of Chinese martial arts.

\section{Competitiveness of Chinese martial arts}

The combination of traditional martial arts and competitiveness derives two terminologies, competitive martial arts and traditional martial arts. In fact, there is no division as competitiveness and tradition in martial arts. Competitive sports was put forward in the eighties of last century, thus to form competitive martial arts, the quickly developed competitive martial arts became obvious gradually and broke the pattern of traditional martial arts. There is no development space for folk martial arts practice, but competitive martial arts could not be agreed, competitive martial arts have something to do with the deterioration of traditional martial arts. If depth analysis is made, it can be found that they don't belong to the same concept at all, and there are also differences in content. Competitive martial arts belong to the inexorable trend of modernization development of martial arts. For example, martial arts are usually used to represent traditional sports. Modern martial arts are formed after blending in western culture. Competitive martial arts need to differ from social martial arts and school martial arts. There is a certain gap in competitiveness between competitive martial arts and traditional martial arts. Competitive martial arts have some standard requirements, and the latter has corresponding competitiveness, which belong to sub-competitive conditions. For martial arts, competitiveness has certain attributes, which are not specific for all martial arts.

\section{Burgeon and depression of Chinese martial art competition}

The core of athletics is competition actually. Yanzi’s Spring and Autumn Annals, the Chinese ancient book of pre-Qin recorded that: "All people with sap have the hearts of competition". The ancient Greek epic Iliad described that people have the thought of being the first forever and fighting 
for surpassing others. These expressions all indicate that China highly praised the idea of competition since ancient, thus to appear the budding competition idea of martial arts gradually. The Commentary of Zuo expressed that the Jin Wengong dreamed to fight with Emperor Cheng of Chu, and the Emperor Cheng of Chu fell on his body and sucked his brains. The translation is that Jin Wengong fights with his opponent in dream and got victory, it is a common competitive pattern. The record in Book of Rites - Kingship showed that there were competitive modes of bare-handed fight at that time. Historical materials can explain that competitive martial arts were firstly seen in period of pre-Qin and the competitive martial arts were in an imperfect stage at that time and it was still under development. There is a expression as follows in Zhuangzi • the View on Sword: "The swords of ordinary people, wearing solid feathers......cut liver and lung” It showed that sword fights were barbaric competitive activities at that time, it had no sports form. The main purpose of competitiveness of martial arts is to defeat opponents, and it is a real battle which can't be broken away from reality. The corresponding cultural spirits without presentation of certain competitive sports belong to the rudiment of competitive martial arts ${ }^{[1]}$.

Competitive martial arts belong to the embodiment of social culture, and it will be restricted by traditional historical culture to some extent. Although competitive martial arts are firstly seen in pre-Qin, competitive martial art sports was not developed smoothly in ancient China. There are two core thoughts in Chinese culture. Confucian and Taoistic theory both can fit the competitive idea of competitive martial arts. The core thoughts of Taoism include "action through inaction". Among them, the nothingness and inaction negate competitive idea fundamentally. Confucian thoughts oppose fair competition, hierarchy theory and social competition. Treasuring bodies and paying attention to filial piety. The above-mentioned thoughts will impact the development of competitive idea of martial arts greatly. So the development of competitive martial arts is vanished gradually, stopping where it should stop in martial arts teaching, thus to form the mode of embodying skills or exercising martial arts, and it is used to replace the form of direct battle. Military strategists pay attention to thoughts and strategies, which will restrict the development of martial art competition. The spiteful martial art competition modes are used for defeating opponents which don't fit basic principles. The literary quotation which is eulogized everywhere in China is Tianji's horse racing.

\section{The enlightenment and exploration of Chinese martial art competition}

Competitive sports were formed in modern times. Competitive ideas were formed due to continual development of economy and led social values. Spenser used biological evolution rules to explain social development phenomenon and built social Darwinism. The fundamental principles for people's survival were formed, which include competition, destroy and survival of the fittest, the weak who impede development should be destroyed, the strong should be remained for survival and provide basis for developing capitalist society. Modern competitive sports were also raised, and the main basis of development is that the spiritual connotation of developing market economy matches with the competitive spirit of competitive sports. People's ideal competition mode is competitive sports. The consciousness of fair competition can be cultivated to some extent through watching and participating in matches. Yan Fu, the modern enlightenment thinker, introduced Spenser's theory in Theory of Natural Selection in detail, he put forward that the basis of evolution is competition, and the idea of sports save country was also formed. Many people put forward national ideas for strengthening the country and provide some power for developing martial art competition. Besides, the disadvantages of traditional martial arts are easy to be exposed with the continuous blending in western competitive sports, which provide some basis and guarantees for competitiveness development of Chinese martial arts ${ }^{[2]}$.

In 1923, the way to change competitive mode of traditional martial arts was "The Chinese National Martial Arts Games” held in Shanghai, which played an important role in developing Chinese martial art competition. In addition, the comprehensive sport events all over the country became the key which marked the development of martial art development. During the $11^{\text {st }}$ Olympic Games, Chinese Martial Arts Performance Team went to Berlin and the world, the rules of martial 
arts competition kept formulating and improving. In the third national games held in 1924 of old China, martial arts were performed as a whole event for first time, basic and easy scoring standard was formed, which were five techniques of hand, eye, body, skill and step. The competition rules were formed through holding two state examinations of martial arts. The limitation of time created the pioneering of confrontation games. The rankings and heavy weight classes were not differentiated in matches. They were only tentative modern competitive sports. The fifth national games perfected competition rules further, the division was made according to event level, weight and so on, and the basic competition patterns of confrontation martial arts were formed. But modern martial art competition was died due to the appearance of war.

\section{Development and Upsurge of Chinese Martial Art Competition}

Since the establishment of new China, the martial art competition ushered in a new development. The sport meeting was held in Tianjin, 1953. Its main performance content was the martial art in fact, which brought new power for the martial art competition. The Competition Rules of Martial Art was issued and implemented in 1959, which referenced to western countries'. It indicated that the martial art competition was formed formally. In order to promote the development and progress of the martial art competition, the a series of skills and trick in Changquan category were formed on the premise of taking example by Chaquan, Huaquan, Paoquan, Hongquan and Huaquan. It was regarded as the basic rules and standards. Afterwards, the Nanquan and Taijiquan were ruled, the dominant forms including Nanquan, Taijiquan and Changquan were formed, the development situated in a new form. The preferential development of the martial art competition limited the development of the traditional martial arts. The exchange meeting in 1979 made the "martial arts heat" form gradually. Many representative competitions were held. The rules of competition were issued gradually in 1980s, which became the direction of the development of the martial art competition. In 2004, relevant national authorities organized to demonstrate fifty-six kinds of power competition rules and competition items for two times, and the Rules of Martial Art Power Competition was formed. After successfully holding the national martial arts competition, the traditional martial arts gradually develop in the direction of the martial art competition. Moreover, in the same year, the word approval martial art competition with the name of Oriental Olympic was held in Zhengzhou, which accelerated Chinese martial art competition to walk up to the world. ${ }^{[3]}$

\section{The Introspection of Chinese Martial Arts Competitiveness}

With continually blending in the western sports culture, the traditional martial arts have formed new forms--competitive martial arts in the process of continuous development. It can promote the development and advance of the martial arts to a certain degree. The sustainable developing traditional martial arts and sports culture globalization can be analyzed from the following three aspects:

\section{The constraint of the western sports culture}

The base of rapidly developing the competitiveness of traditional martial arts is the Western sport competitive idea and the theory of evolution. The developing source of western culture is the ancient Greek culture. Its main core is analytic thinking, which promotes the competition model of the western competitive sports of the martial arts competitive events. The traditional martial arts formed according to Chinese traditional culture focus on systematic thought, its fundamental characteristic is both the cultivation of body and martial arts. The westernization of Chinese traditional martial arts are the mode of constantly blending in Western thinking and breaking the traditional thinking. It can form the fighting-and-dancing-well according to the traditional martial arts competitive thinking. But the development of the fighting-and-dancing-well can be limited by fair competition. It can change the thinking model and prompt the traditional martial arts to form 
two kinds of sports system, which including free combat and routines, thus to form different levels of free combat competition and different forms of routines competition. It can separate free combat and routines, Ensure their independent without losing unification. It's right for blending the international competitive Olympic Games into the traditional martial arts and separating the free combat and routines, which conform to the western ideas and fair idea. But it not only has one model, the traditional martial arts need to establish the model according to its development when analyzing, thus to ensure sustainable development. ${ }^{[4]}$

\section{The variation of technique styles}

The combined action of Olympic Games and competition make the rapid developing martial arts competition causes great influence on social martial arts activities. The traditional models of martial arts have not been approved gradually. It starts to give up the traditional models and set up the martial arts competition. When performing the traditional martial arts before, ornamental value got most of the attention. A lot of executed difficult Changquan movements were applied when performing the routines. But the formed performing effects with different styles lost the characteristics of traditional martial arts. Most of the movements in many performance ways except beginning form and closing form are the movements of competitive martial arts. Martial arts schools improve the strength of teaching the competitive martial arts when teaching the traditional martial arts. The style of martial arts has reduced gradually with the action of the competitive martial arts. It is impracticable to imitate competitive martial arts simply, and it will go against the development of the competitive martial arts. Therefore, we should rethink continually for prompting the competitive martial arts on the right path.

\section{The Withering Fitness Domain}

The main basis of the development of competitive martial arts is contest system, which has certain significance in improving competitive level and promoting nationalization development. Developing competitive martial arts can promote the progress of traditional martial arts to some extent. The new normative contest routines, martial arts stages, contests become highlights gradually. In aspect of public physical training, it keeps changing people's life styles, and traditional martial arts provide great help for the development of China. It is gradually replaced by some pleasant and relaxed, easy-to-learn western forms due to dull practice, tedious contents and so on. If the modernized transformation step of Chinese martial arts can't be improved, it's very likely that there is only Tai Ji left in martial arts in the future. During the process of continual researches, crisis in developing martial arts is hidden. With the continual development of modern sports, it still loses the position in fitness gradually based on getting certain effects in developing traditional martial arts, new changes and choices need to be made.

\section{Outlook on competitiveness of Chinese martial arts}

The main charms of traditional martial arts are rich, colorful and varied Chinese boxing. In order to maintain the charms of traditional martial arts, propagation and communication needs to be quickened and technology system needs to be standardized. Affirming, developing and inheriting technologies with obvious technical features, rich cultural connotation and good for developing health, changing people's life styles, promoting easy-to-learn technical motions, satisfying social development and actual routines which are required by people on entertainment and health. The core of modernized development on Chinese traditional martial arts is competitiveness. Martial arts competitiveness is implemented according to martial arts competition norms developed by the West. Western sports form can promote the development of modernized martial arts to some extent, and it also limits the development of modernized martial arts. Competitive free combat and competitive routines are formed under the premise of Chinese traditional martial arts. The main form of development and improvement of competitiveness is western boxing and gymnastics. Beijing held Olympic Games in 2008, and it promoted competitive free combat and competitive routines to be the events of Beijing Olympic Games officially. After a lot of practice, it can be found that it is not an 
effective martial arts competition mode which fits the national condition if blindly pursuing for westernization of martial arts competitiveness. The competitiveness of Chinese martial arts needs to make fully analysis based on western patterns, find local elements which fit national conditions and build polybasic martial arts competition patterns, thus to promote the development and progress of transformation of Chinese martial arts competition and provide basis for reaching out to the world ${ }^{[5]}$.

\section{Conclusion}

In a word, the competitiveness transformation of Chinese martial arts will become the inexorable trend of development in the future gradually. It is also the important period which adapts era development, but it not belongs to the actual affiliation of Chinese martial arts. From the aspect of developing culture, it can be found that Chinese martial arts belong to specific sports embodiment of local culture. The track will be deviated if there is no basis of local culture. The competitiveness transformation of Chinese martial arts lays the foundation of its development.

\section{Acknowledgment}

This paper is the routine subject in 2015 of Shanxi Sports Bureau, which name is Brand Building and Communication of Martial Art Sanda Commercial Matches_Taking “China Kung Fu” of Shanxi TV as an example, Subject No. 15102.

\section{References}

[1] Zhang Changnian, Wang Gang. Analysis on the Causes of Cultural Transformation of Chinese Wushu in Its Present Development. Journal of Capital University of Physical Education and Sports, 2014, 26(2): 11-15.

[2] Lu Xiaohei, Tang Meiyan. "Reward the same"and "punish the different": the propagation rules for cross -culture communication of Chinese martial arts. Journal of Shandong Institute of Physical Education and Sports, 2015(2): 58-63.

[3] Yuan Weijun, Wu Song. Chinese Martial Arts' Return to Public Life: from “a Sport” to "an Art”. Journal of Chengdu Sport University, 2011, 37(11): 37-40.

[4] Shen Guoqing. Development of Chinese Wushu from 2008 to 2012. Journal of Wuhan Institute of Physical Education, 2013, 47(11): 59-64.

[5] Han Zheng, Wang Gang. Historical Changes from "Grass-Roots Culture" to "Symbolic Culture" of Chinese Martial Arts since the Modern Times. Journal of Shandong Institute of Physical Education and Sports, 2014(6): 50-55. 